\title{
Optimizing Process-Oriented Translator Training Using Freeware and FOSS Screen recording Applications
}

Erik Angelone

Kent State University, USA

\section{Fundamentals of Process-oriented Translator Training:}

\subsection{Definitions, Models and Descriptions}

As an empirically-drive pedagogical approach, process-oriented translator training, in a broad sense, focuses on enhancing learner awareness of how one translates. This overarching notion of 'how' can be approached from numerous, interrelated perspectives, including awareness of such phenomena as the nature of problems encountered and subsequent problem-solving tendencies (Angelone 2013a), segmenting behavior (Dragsted 2005; Hansen 2006), information retrieval tendencies (Alves and Liparini Campos 2009), general workflow patterns (Pym 2009), and cognitive ergonomics (Ehrensberger-Dow and Massey 2014). By deliberately shifting away from the translation product in and of itself as a relatively shallow snapshot of student performance, process-oriented training sets out to foster awareness of how this product was reached in the first place as a result of decision-making and strategy execution at the three fundamental loci of comprehension, transfer, and production. Given the fact that translation, at its very core, is a higher order cognitive task, process-oriented training approaches draw from numerous problem-solving models established within the cognitive process research community, such as that found in Figure 1:

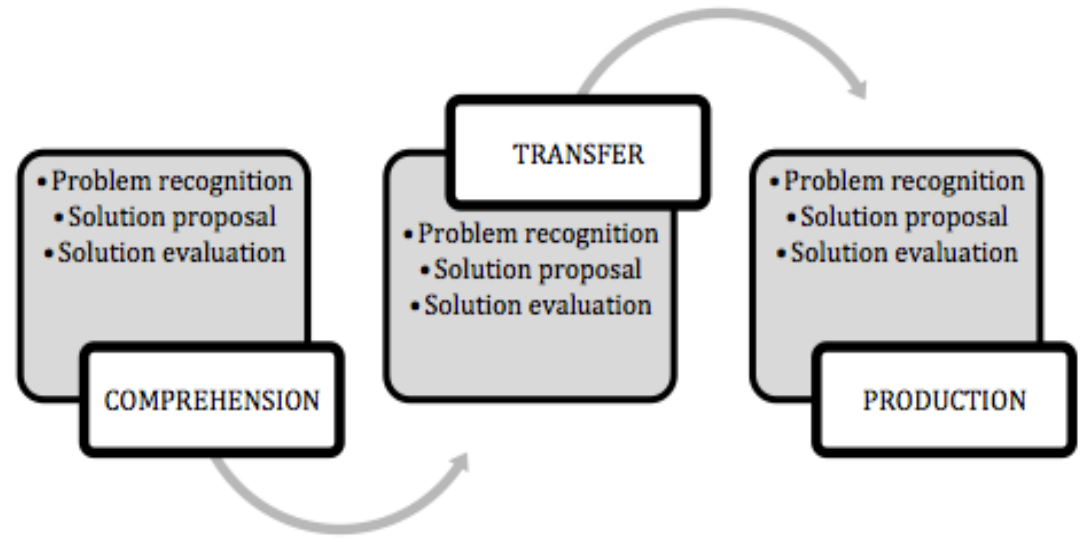

Figure 1: Loci and behaviors of problem-solving in translation (Angelone 2010). 
Problem recognition involves knowledge assessment in relation to a given problematic aspect of the task at hand. There tends to be a breakdown in the natural flow of translation, with the most directly observable indicator thereof being an extended pause in translation activity. Solution proposal behavior involves strategy execution in response to the given problem, as indicated first and foremost by various forms of information retrieval. Whereas solution proposal concerns itself with generating options, solution evaluation involves narrowing them down in line with situational constraints. This is very much geared towards choosing among options, as driven by contextual factors and deliberate decision-making in light of them. All three of these behaviors (problem recognition, solution proposal, solution evaluation) can occur at any one the three loci (comprehension, transfer, production), often in a bundled, sequential fashion (Angelone and Shreve 2011: 120). Taken holistically, most directions in process-oriented translator training target some dimension of this particular problem-solving model.

\subsection{Methods and Approaches}

Process-oriented training began in earnest in the 1990s, when Kiraly (1995) called on trainers to shape a curriculum around optimal strategies, decisions, and behaviors exhibited by successful professional translators in authentic contexts. For the better part of that decade, translation process research and resultant pedagogical practices were driven by three primary methods: 1) Integrated Problem and Decision Reporting logs (Gile 2004), 2) think-aloud protocols (TAPs), and 3) keystroke logging. An IPDR log is a student-created running list of all problems encountered while translating along with correlating documentation of problem-solving strategies, rationales, and solutions used in addressing them. Creation requires students to temporarily break away from the translation task at hand to document content, which usually appears in tabular form in a separate document. IPDR logs are useful in generating whole-class discussion of problem-solving strategies in relation to a given text. However, the documented content is not always an entirely accurate reflection of the problems students faced, as revealed through mismatches between reported problems and actual errors that appear in corresponding translation products. This may by the result of still underdeveloped student self-reporting of problems, with problems tending to either go unnoticed or be defined in an incomprehensive fashion.

A think-aloud protocol consists of audio documentation of articulations representing thought processes that transpire over the course of translation. Students are instructed to engage in consistent, continuous verbalization in a relatively freeform manner. Retrospective analysis of recorded audio content can reveal problems and problem-solving tendencies in the form of extended 
periods of silence, direct/indirect articulation, or a variety of speech disfluencies. Some students might feel uncomfortable with having to simultaneously translate and articulate what is going through their minds, not to mention cognitively overtaxed by this dual task. As a result, it is advisable to keep the length of the texts to be translated short (200 words or less).

Towards the end of the 1990s, in response to documented shortcomings of translation logs and TAPs, keystroke logging became a methodology of choice for process-oriented training (cf. Hansen 2006). Here, a software application records all keystrokes, mouse clicks, deletions, and instances of cursor repositioning for purposes of retrospective analysis. Additionally, keystroke loggers document valuable temporal data, such as pause intervals and uninterrupted text segment durations, both windows to problems and problem-solving. The efficacy of keystroke logging as a lens to translation processes is evidenced by the fact that it is still very much a method of choice in the research community. Nevertheless, as depicted in Figure 2, from the student's perspective, making sense of highly granular data for purposes of self-reflection on problem-solving might be an onerous task.

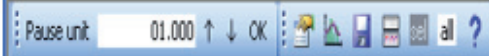

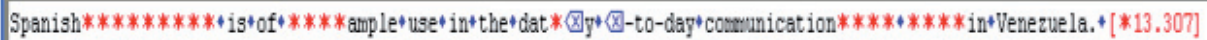

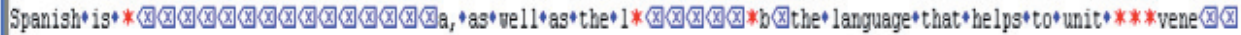

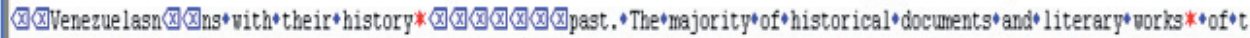

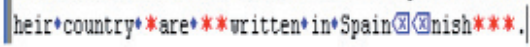

Figure 2: Keystroke log output from Translog.

Over the past five or so years, a second generation of process-oriented translator training has come into existence, driven by two new methods on the cognitive process research front: 1) eye-tracking, and 2) screen recording. Eye-tracking technology, which documents visual attention data in the form of heat maps and gaze plots, holds great potential in helping trainers and trainees glean insight as to where students look on the screen and for how long when encountering and solving problems. To date, we have not seen much (if any) research on pedagogical applications of eye-tracking due to the high costs of existing commercial tools, but with the advent of open source eye-tracking applications, such as Opengazer (www.inference.phy.cam.ac.uk/ opengazer), this may very well change in the near future.

Screen recording is made possible by a software application that captures all on-screen activity that occurs over the course of task completion, documenting such phenomena as extended pauses, information retrieval 
(triggers and types of resources utilized), the textual level of target text generation, and revision tendencies. As is the case with TAPs, keystroke log output, and the visual attention data made available through eye-tracking, when using screen recordings, reflection on various aspects of the translation task takes place during a retrospective session. Unlike eye-tracking, screen recording has gained firm footing in recent years as an optimal tool for processoriented training, particularly with the advent of freeware and open source options. Reasons for this trend will be outlined in the next section. Table 1 below provides an overview of some of the advantages and disadvantages associated with the five process-oriented training methods discussed in this section.

Table 1. Process-oriented training methods

\begin{tabular}{|l|l|l|}
\hline Method & \multicolumn{1}{|c|}{ Major advantage } & \multicolumn{1}{|c|}{ Major disadvantage } \\
\hline $\begin{array}{l}\text { Integrated Problem and } \\
\text { Decision Reporting logs } \\
\text { (Gile 2004) }\end{array}$ & $\begin{array}{l}\text { Personalized content/ } \\
\text { User-friendliness }\end{array}$ & $\begin{array}{l}\text { Mismatch between perceived } \\
\text { problems documented and actual } \\
\text { errors in the product }\end{array}$ \\
\hline $\begin{array}{l}\text { Think-aloud protocols } \\
\text { (TAPs) }\end{array}$ & $\begin{array}{l}\text { Heightened cognitive } \\
\text { focus when translating } \\
\text { short (<200-word) texts }\end{array}$ & $\begin{array}{l}\text { Cognitive/physical exhaustion from } \\
\text { having to articulate all thought } \\
\text { processes }\end{array}$ \\
\hline Keystroke logging & $\begin{array}{l}\text { Temporal data provides } \\
\text { clear insight into } \\
\text { allocation of cognitive } \\
\text { effort }\end{array}$ & $\begin{array}{l}\text { Students may lose sight of the } \\
\text { "bigger picture" due to very granular } \\
\text { data }\end{array}$ \\
\hline Eye-tracking & $\begin{array}{l}\text { Multiple triangulated } \\
\text { levels of visual attention } \\
\text { data (heat maps, gaze } \\
\text { plots, saccades) } \\
\text { precision }\end{array}$ & $\begin{array}{l}\text { Lack of portability/universality/ } \\
\text { ecological validity }\end{array}$ \\
\hline Screen recording & $\begin{array}{l}\text { Highly visual rendition of } \\
\text { problem solving results } \\
\text { in heightened awareness } \\
\text { of problems. (Angelone } \\
\text { 2013a) }\end{array}$ & $\begin{array}{l}\text { Thoughts underlying activity not } \\
\text { always discernable = need for } \\
\text { immediate retrospection post task } \\
\text { completion }\end{array}$ \\
\hline
\end{tabular}

\section{Screen Recording as a Preferred Tool}

There are a number of reasons why trainers might want to turn to screen recording as an optimal tool for freeware and FOSS-driven process-oriented training. Firstly, recent empirical research has suggested that screen recording, when compared with IPDR logs and TAPs as diagnostic protocols for 
documenting student translation performance, is more efficacious in the domains of problem awareness and error mitigation (Angelone 2013a; Shreve et al. 2014). In a series of studies, students created logs, TAPs, and screen recordings in conjunction with various translation tasks and were asked to utilize the respective process protocol as a diagnostic tool of sorts to make any necessary changes to the corresponding translation products. When screen recordings were utilized for this purpose, fewer errors ultimately remained in the revised texts for the vast majority of students than when the other protocol types were used. This held true in tasks involving both self-revision and otherrevision. The highly visual medium and manner of reflection would seem to potentially make problems more salient. This particularly holds true in light of the fact that students watch their performance as it originally unfolded in a very natural context. As previously mentioned, they do not have to do anything they would not otherwise already be doing while translating besides pressing record and stop. They do not have to work in an otherwise foreign interface. They do not have to make sense of numbers generated by an overly complicated analytic software application. They can engage in analysis from the comfort of their own homes on their own computers, thanks to cross-platform options. At the click of a mouse, they can fast-forward, rewind, and pause videos so that analysis transpires at their own preferred pace in a learner-centered fashion that is much less dependent on the trainer.

When screen recording technology was first integrated for research and training purposes, options were somewhat limited, with the vast majority of initiatives relying on Camtasia Studio, a proprietary application launched by the company TechSmith in 2002. At the time of writing, a single user license at education pricing rates costs \$179 USD. Over the past decade, freeware and open source alternative options have entered the scene, as outlined below in Tables 2 and 3 . Screen recording has evolved to become truly universal in the sense that it is not restricted to any one operating system/platform, output format, or programming language. Trainers and trainees should be able to find an application that best meets their potentially unique needs and preferences in terms of technical requirements and features. It is important to note that the FOSS (free and open source) options offer more or less the same level of functionality and range of features as their commercial counterparts. Quality is in no way sacrificed.

\subsection{Screen Recording Features from a Training Perspective}

Tables two and three below provide information on a selection of free screen recording applications, with variation at the levels of classification (freeware, freemium, or open source) and operating system (Windows, Mac OS, or Linux). These six applications, rather than representing an exhaustive list of 
all that is available, were selected for inclusion based on a level of functionality and range of features that compare with Camtasia Studio as a commercial application benchmark. A brief overview of the various features with an eye towards pedagogical applications in the context of processoriented translator training will be followed by descriptions of concrete learning activities.

\section{Audio Recording (AUDREC)}

This feature enables translators to capture audio documentation of their problems, problem-solving strategies, and general thought processes in the form of recorded articulations. The obtained audio data, in essence a TAP, parallels visual data representing on-screen activity, thereby providing a more granular depiction of translation processes. From the perspective of problem awareness training, students could be encouraged to focus in on such things as direct/indirect articulation of problems, extended periods of silence in articulation, and various speech disfluencies in retrospective analysis of their work.

\section{Webcam Recording (WEBCAM)}

With this feature, translators and translator trainers can obtain documentation of things like facial expressions, body language, and physical reactions in a broad sense in conjunction onscreen activity. In this sense, webcam data can be regarded as the non-verbal counterpart to the verbal data captured through audio recordings, adding another layer of granularity to the documentation and subsequent analysis of translation processes.

\section{Scheduled Recording (SCHED)}

This feature provides the option of starting and stopping recording at pre-set times and for a pre-set duration. If, for example, students or trainers want to examine how translation processes vary at different points of the task as it progresses (i.e., what do students do for the first ten minutes or the last ten minutes,), this feature could provide such snapshots for retrospective analysis. Obtaining such snapshots might also be interesting in documenting translator style and how this style might vary in situations involving timed vs. untimed tasks.

\section{Real-time Pausing (PAUSE)}

With this feature, translators can pause recording and continue at a later time, implying that there wouldn't be a need to complete the entire translation task in one sitting. This becomes particularly helpful in the context of lengthy texts, where the translator would likely be more inclined to take frequent breaks. This feature would also be helpful in situations where the trainer or trainee is 
looking for documentation of only a specific aspect of the translation task, such as information retrieval tendencies. Everything else could be filtered out of the screen recording using real-time pausing.

\section{Post-editing (EDIT)}

This feature enables cutting, merging, or adding frames within a given screen recording after it has been created. This gives the trainer the option of creating montages to highlight such things as different ways of approaching the same problematic text passage or the execution of the same particularly efficacious problem-solving strategy at different locations in the task.

\section{Annotation (ANNOT)}

The annotation feature gives students and trainers the option of inserting various comments, such as documentation of observations, explanations underlying various strategies, etc., directly into the created screen recording. Depending on the application being used, the annotation may take the form of text, graphics, or even embedded videos.

\section{URL-based Sharing (SHARE)}

Screen recordings, particularly those representing longer translations (upwards of an hour), can be quite large in terms of file size, making sharing via email or e-learning platforms potentially problematic. The screencast sharing feature basically stores the recordings in an on-line repository that can then be accessed by others via designated urls. This is a nice way of sharing files based on permission settings and overcomes space limitations associated with other options.

\section{Unlimited Recording Length (LNGTH)}

Some screen recording applications have a set maximum recording time before automatically shutting off. Others enable recording videos of unlimited length, implying fewer restrictions on variables such as text length and difficulty, not to mention one less thing for trainers or trainees to worry about in an attempt to preserve ecological validity. 
Table 2. A selection of screen recording options

\begin{tabular}{|l|l|l|l|l|}
\hline \multicolumn{1}{|c|}{ Application } & \multicolumn{1}{|c|}{ Publisher } & \multicolumn{1}{|c|}{ Source } & \multicolumn{1}{c|}{ Classification } & \multicolumn{1}{c|}{ OS } \\
\hline $\begin{array}{l}\text { Blueberry } \\
\text { Flashback Express }\end{array}$ & $\begin{array}{l}\text { Blueberry } \\
\text { Software }\end{array}$ & http://www.bbsoftware.co.uk/ & Freeware & Windows \\
\hline CamStudio & CamStudio & http://camstudio.org/ & Open Source & Windows \\
\hline EZVid & EZVid, Inc. & http://www.ezvid.com/ & Freeware & Windows \\
\hline $\begin{array}{l}\text { Open Broadcaster } \\
\text { Software }\end{array}$ & $\begin{array}{l}\text { obs.jim@ } \\
\text { gmail.com }\end{array}$ & https://obsproject.com/ & Open Source & $\begin{array}{l}\text { Windows } \\
\text { Mac OS } \\
\text { Linux }\end{array}$ \\
\hline Quicktime & Apple Inc. & $\begin{array}{l}\text { https://www.apple.com/quickt } \\
\text { ime/download/ }\end{array}$ & Freemium & $\begin{array}{l}\text { Windows } \\
\text { Mac OS }\end{array}$ \\
\hline recordMydesktop & $\begin{array}{l}\text { Martin } \\
\text { Nordholts } \\
\text { fttp://recordmydesktop.source } \\
\text { forgent/ }\end{array}$ & Open Source & Linux \\
\hline
\end{tabular}

Table 3. A comparison of applications by features

\begin{tabular}{|l|c|c|c|c|c|c|c|c|}
\hline Application & AUDREC & WEBCAM & SCHED & PAUSE & EDIT & ANNOT & SHARE & LNGTH \\
\hline $\begin{array}{l}\text { Blueberry } \\
\begin{array}{l}\text { Flashback } \\
\text { Express }\end{array}\end{array}$ & + & + & + & - & + & - & + & + \\
\hline CamStudio & + & + & - & + & - & + & - & + \\
\hline EZVid & + & + & - & + & + & + & + & $-(45 \mathrm{~min})$ \\
\hline $\begin{array}{l}\text { Open } \\
\text { Broadcaster } \\
\text { Software }\end{array}$ & + & + & - & + & + & + & + & + \\
\hline Quicktime & + & - & - & - & + & - & - & + \\
\hline $\begin{array}{l}\text { recordMy } \\
\text { desktop }\end{array}$ & + & - & - & + & - & - & - & + \\
\hline
\end{tabular}

\section{Pedagogical Approaches and Learning Activities Using Screen Recording}

Given the constellation of features outlined above, screen recording has proven to be a versatile application for purposes of process-oriented translator training. This section will describe a series of screen recording-based learning and assessment activities to facilitate learning along these lines.

\subsection{Self-awareness of Problems}

As mentioned above, empirical research on student problem-solving has indicated a tendency for problems to often go unnoticed (Göpferich 2009). Furthermore, what students assume to be problematic often represents only a narrow scope of what is truly problematic from the perspective of errors that 
result in their translations. Having learners create screen recordings in conjunction with their translations establishes empirical grounds for diagnostic self-reflection and a mechanism for training problem awareness at a much more granular level than possible when examining the product alone. Prior to having students engage in self-reflection, it is paramount for trainers to guide them through the process and introduce various focal points, starting with potential problem indicators embedded in the screen recordings. Primary problem indicators include extended pauses in screen activity, instances of information retrieval, and revisions, among others. When analyzed empirically by students on a regular basis and across a variety of translation tasks, these are the kinds of phenomena that can yield a more holistic understanding of the nature of problems and problem-solving.

If students have the opportunity to submit drafts of a given translation, analysis of screen recordings in this capacity can serve as an important error detection editing stage prior to re-submission. Students could also be asked to write up a reflection on their problems and problem-solving tendencies using the following questions as prompts: 1) What tended to pose problems based on observed occurrences of extended pauses in screen activity? 2) How would you describe the nature of these problems from the perspectives of textual level (lexis, syntax, stylistic) and locus (comprehension, transfer, production)? 3) Which resources did you tend to utilize in addressing the problems and why? 4) In retrospect, was there anything that surprised you about the problems you encountered and the manner in which you went about solving them? 5) In retrospect, would you have done anything differently? Why? The documentation of these observations could serve as formal assignments or as a springboard for in-class discussion during workshopping sessions. Given the annotation feature described above, observations could be documented in the screen recording environment itself, eliminating the need for a different (separate) application for this purpose. Assignments could be submitted using the url-based sharing application inherent to many screen recording tools. Free and open source applications, in particular, have greatly advanced this 'all-in-one' approach, where student and instructor comments can be directly embedded in screen recordings, making file management and transfer that much easier.

\subsection{Re-tracing Errors in the Product through the Processes}

When it comes to feedback on their performance, students often have little more than marked-up errors in their translations to go on. These markings likely provide them with quantitative insight regarding the types of errors they make, yet often shed no light on why these errors may have occurred in the first place from a process-oriented perspective. For example, an error code 
might reveal to the student that a terminology error has occurred, but he or she might not know why. Was it a result of inaccurate information retrieval? Was it a result of simply not knowing what the term means? Did he or she have the right term first and then go back and erroneously change it during a revision stage? Was the term's usage cross-checked using parallel texts? Did the terminological error co-occur with extended pauses to signal a potential problem? Screen recording documentation would enable the student to retrace the error and answer these questions in obtaining a clearer insight into its nature, transcending beyond the textual level alone, as indicated in the mark-up. As a very basic learning activity, students could be asked to re-trace all of the errors in their translations and comment on why the errors may have occurred based on what they observe in their screen recordings. This form of

self-assessment adds a much-needed procedural dimension to helping students understand the nature of errors.

\subsection{Watching and Learning from Virtual Professionals}

Screen recording can also be an effective way to introduce students to the problem-solving tendencies of professional translators. This can best be accomplished by having professionals create screen recordings while translating the very same texts that students will be asked to translate, establishing grounds for comparative process analysis (Angelone 2013b). Students could be asked to focus on similarities and differences, at a very basic level, thereby enhancing awareness of multiple problem-solving pathways. Trainers could use this comparative approach as a way of modeling best practices from an expertise perspective, where students are asked to comment on the behaviors and strategies of particularly successful professionals. Additionally, students could be asked to comment on where the professionals seem to struggle, or where their own problem-solving approaches could be regarded as more efficacious than those of the professionals. This latter activity can be particularly helpful in motivating learners and boosting their self-confidence. Additionally, it presents the real world of professional translation as being within reach.

\subsection{Workshopping the Process}

In a product-oriented training environment, a common pedagogical approach involves comparative analysis of translation products on a sentence-bysentence basis. Screen recording enables an approach that focuses on how TT solutions were generated in the first place, also in a comparative fashion. Using the aforementioned editing feature, trainers can create collages representing multiple problem-solving approaches in conjunction with select text passages. Instead of reading multiple target text options on screen, 
students would watch multiple target text options emerge in real time. This learning activity could be centered around an examination of what unfolds in conjunction with text passages that the trainer regards as being 'rich points' (PACTE 2011: 38), or predicted sources of disturbance (Hansen 2006). Alternatively, depending on how much lead time is available prior to in-class workshopping, the trainer could create collages based on observed, patterned problems. This would be conducive in situations where there is a potential mismatch between passages the trainer assumes will be problematic and passages that actually prove to be problematic based on evidence documented via screen recording.

\subsection{Snapshots of Performance for Process-oriented Assessment}

Formal assessment of translation using screen recording technology is a domain in which a vast amount of research is still waiting to be done. At the Zurich University of Applied Sciences, screen recording is being utilized in the context of assessing borderline entrance translation exams (Massey and Ehrensberger-Dow 2013). Given the fact that the translation product represents a somewhat limited view of student performance, taking a closer look at underlying processes might provide a more accurate (or at least more granular) reflection on student performance patterns (and potential) on the whole. That being said, given the length of screen recordings, holistic analysis of screen recordings in conjunction with each and every translation becomes less of an option for the individual trainer, particularly in the context of a higher enrollment class. To compensate for this, using the scheduled timer feature, trainers can utilize screen recording to capture a shorter representative sample of a larger translation task to analyze in conjunction with grading of the translation product. Quantitative metrics currently are not in place to guide process-oriented grading as such. In this case, ungraded feedback on processes can serve as an ideal complement to a concrete grade/letter score assigned to the product, even if based on only ten or so minutes of content.

\section{New Horizons through a Freeware/FOSS Lens}

Given the still predominately product-oriented focus of translator training and assessment (Dam-Jensen and Heine 2009: 1) and the fact that extensive feedback in the world of professional translation is seldom present, both students and professionals rely to a large extent on self-assessment in gauging their performance. In this sense, screen recording, as a processoriented self-assessment tool, should be on equal footing with other freeware and FOSS applications constituting assistive translation workbenches, such as tuxtrans (Sandrini 2007) or CasMaCat (Koehn et al. 2012). The CasMaCat 
open source workbench is already geared towards 'automatic analysis of translator behavior' (Alabau et al. 2013: 105) thanks to a logging and replay component based first and foremost on eye-tracking and keystroke logging technology. The inclusion of a screen recording component would likely enhance user-friendliness from the student's and trainer's (as opposed to the researcher's) perspectives in particular.

Interestingly, unlike what is the case for such CAT applications as translation memories and terminology management systems where industry-leading commercial options have emerged, there is no commercial screen recording benchmark against which FOSS and freeware options would need to compete. This gives each individual user (whether trainer, trainee, or professional) the freedom to pick and choose from a variety of screen recording options that best suit his or her unique needs and preferences without feeling forced into choosing a set industry standard and without having to worry about licensing or budgetary constraints.

In summary, as a CAT tool whose potential as a vehicle for enhancing process awareness is just now being realized in academic contexts, screen recording truly embraces portability, flexibility, and opportunities for customization envisaged by open source as a development model. It is hoped that the ideas presented in this paper will further motivate trainers, trainees, professional translators, and the language industry at large to explore how freeware and FOSS screen recording can be integrated to enhance translation pedagogy.

\section{References}

Alabau, V., Bonk, R., Buck, C., Carl, M., Casacuberta, F., García-Martínez, M., González, J., Koehn, P., Leiva, L., Mesa-Lao, B., Ortiz, D., Saint-Amand, H., Sanchis, G. and Tsoukala, C. (2013) CASMACAT: An Open Source Workbench for Advanced Computer Aided Translation. The Prague Bulletin of Mathematical Linguistics 100, 101-112.

Alves, F. and Liparini Campos, T. (2009) Translation Technology in Time: Investigating the Impact of Translation Memory Systems and Time Pressure on Types of Internal and External Support. In S. Göpferich, A.L. Jakobsen, and I. Mees (eds.) Behind the Mind. Methods, Models and Results in Translation Process Research. Copenhagen: Samfundslitteratur Press, 191-218.

Angelone, E. (2013a) The impact of process-protocol self-analysis on errors in the translation product. Translation and Interpreting Studies 8(2), 253-271.

Angelone, E. (2013b) Watching and Learning from 'Virtual Professionals': Utilizing Screen Recording in Process-Oriented Translator Training. In D. Kiraly, S. Hansen-Schirra and K. Maksymski (eds.) New Prospects and Perspectives for Educating Language Mediators. Tübingen: Günther Narr, 139-155. 
Angelone, E. (2010) Uncertainty, uncertainty management and metacognitive problem solving in the translation task. In G. Shreve and E Angelone (eds.) Translation and Cognition. Amsterdam: John Benjamins, 17-40.

Angelone, E. and Shreve, G. (2011) Uncertainty Management, Metacognitive Bundling in Problem-Solving, and Translation Quality. In S. O'Brien (ed.) Cognitive Explorations of Translation. London/ New York: Continuum, 108-130.

Dam-Jensen, H. and Heine, C. (2009) Process Research Methods and Their Application in the Didactics of Text Production and Translation. trans-kom 2(1), 1-25.

Dragsted, B. (2005) Segmentation in translation - differences across levels of expertise and difficulty. Target 17(1), 49-70.

Ehrensberger-Dow, M. and Massey, G. (2014) Cognitive Ergonomic Issues in Professional Translation. In J. Schwieter and A. Ferriera (eds.) The Development of Translation Competence: Theories and Methodologies from Psycholinguistics and Cognitive Science. Newcastle upon Tyne: Cambridge Scholars Publishing, 58-86.

Gile, D. (2004) Integrated problem and decision reporting as a translator training tool. JoSTrans 2, 2-20.

Göpferich, S. (2009) Towards a model of translation competence and its acquisition: the longitudinal study TransComp. In S. Göpferich, A.L. Jakobsen, and I. Mees (eds.) Behind the mind: Methods, models and results in translation process research. Copenhagen: Samfundslitteratur Press, 11-37.

Hansen, G. (2006) Retrospection Methods in Translator Training and Translation Research. The Journal of Specialised Translation 5, 2-41.

Koehn, P., Alabau, V., Carl, M., Casacuberta, F., García-Martínez, M., González-Rubio, J., Keller, F., Ortiz-Martínez, D., Sanchis-Trilles, G. and Germann, U. (2012) CasMaCat: Cognitive Analysis and Statistical Methods for Advanced Computer Aided Translation. Available at: http://www.casmacat.eu [Accessed 27 June 2015].

Kiraly, D. (1995) Pathways to Translation: Pedagogy and Process. Kent, Ohio: Kent State University Press. Massey, G. and Ehrensberger-Dow, M. (2013) Evaluating translation processes: opportunities and challenges. In D. Kiraly, S. Hansen-Schirra, and K. Maksymski (eds.) New Prospects and Perspectives for Educating Language Mediators.

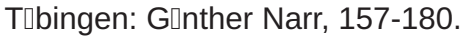

PACTE Group. (2011) PACTE Translation Competence Model: Translation Project and Dynamic Translation Index. In S. O'Brien (ed.) Cognitive Explorations of Translation. London/ New York: Continuum, 30-56.

Pym, A. (2009) Using Process Studies in Translator Training: Self-discovery through Lousy Experiments. In S. Göpferich, F. Alves, and I. Mees (eds.) Methodology, Technology and Innovation in Translation Process Research. Copenhagen: Sammfundslitteratur Press, 135-156.

Sandrini, P. (2007) tuxtrans GNU/Linux Desktop for Translators. Available at: http://www.uibk.ac.at/tuxtrans/ [Accessed 23 June 2015].

Shreve, G., Angelone, E. and Lacruz, I. (2014) Efficacy of Screen Recording in the OtherRevision of Translations: Episodic Memory and Event Models. MONTI 1, 225- 245. 
